\title{
D-BLAST OFDM with Channel Estimation
}

\author{
Jianxuan Du \\ School of Electrical and Computer Engineering, Georgia Institute of Technology, Atlanta, GA 30332-0250, USA \\ Email:jxdu@ece.gatech.edu \\ Ye (Geoffrey) Li \\ School of Electrical and Computer Engineering, Georgia Institute of Technology, Atlanta, GA 30332-0250, USA \\ Email: liye@ece.gatech.edu
}

Received 28 January 2003; Revised 26 September 2003

\begin{abstract}
Multiple-input and multiple-output (MIMO) systems formed by multiple transmit and receive antennas can improve performance and increase capacity of wireless communication systems. Diagonal Bell Laboratories Layered Space-Time (D-BLAST) structure offers a low-complexity solution for realizing the attractive capacity of MIMO systems. However, for broadband wireless communications, channel is frequency-selective and orthogonal frequency division multiplexing (OFDM) has to be used with MIMO techniques to reduce system complexity. In this paper, we investigate D-BLAST for MIMO-OFDM systems. We develop a layerwise channel estimation algorithm which is robust to channel variation by exploiting the characteristic of the D-BLAST structure. Further improvement is made by subspace tracking to considerably reduce the error floor. Simulation results show that the layerwise estimators require $1 \mathrm{~dB}$ less signal-to-noise ratio (SNR) than the traditional blockwise estimator for a word error rate (WER) of $10^{-2}$ when Doppler frequency is $40 \mathrm{~Hz}$. Among the layerwise estimators, the subspace-tracking estimator provides a $0.8 \mathrm{~dB}$ gain for $10^{-2}$ WER with $200 \mathrm{~Hz}$ Doppler frequency compared with the DFT-based estimator.
\end{abstract}

Keywords and phrases: MIMO, OFDM, channel estimation.

\section{INTRODUCTION}

Multiple-input and multiple-output (MIMO) systems formed by multiple transmit and receive antennas are under intense research recently for its attractive potential to offer great capacity increase. Space-time coding, proposed in [1], performs channel coding across the space and time to exploit the spatial diversity offered by MIMO systems to increase system capacity. However, the decoding complexity of the space-time codes is exponentially increased with the number of transmit antennas, which makes it hard to implement real-time decoding as the number of antennas grows. To reduce the complexity of space-time based MIMO systems, diagonal Bell Laboratories layered space-time (D-BLAST) architecture has been proposed in [2]. Rather than try to optimize channel coding scheme, in D-BLAST architecture, the input data stream is divided into several substreams. Each substream is encoded independently using one-dimensional coding and the association of output stream with transmit antennas is periodically cycled to explore spatial diversity.

Orthogonal frequency division multiplexing (OFDM) systems have the desirable immunity to intersymbol interference (ISI) caused by delay spread of wireless channels. Therefore, the combination of D-BLAST with OFDM is an attractive technique for high-speed transmission over frequency- selective fading wireless channels. As in [3], when combining D-BLAST structure with OFDM, we implement the spacetime structure in space-frequency domain to avoid decoding delay. To decode each layer, channel parameters are used to cancel interference from detected signals and suppress interference from undetected signals to make the desired signal as "clean" as possible. Therefore, estimation of channel parameters is a prerequisite for realizing D-BLAST structure and to a great extent determines system performance. In this paper, we investigate D-BLAST OFDM systems and address the channel estimation problem.

DFT-based least-square (LS) channel estimation for MIMO-OFDM systems and simplified estimation algorithm using parallel interference cancellation have been addressed in $[4,5]$, respectively. For D-BLAST OFDM, we propose a layerwise LS channel estimator that exploits the characteristics of the system structure by updating channel parameters after each layer is detected so that later layers in the same OFDM block can be detected with more accurate channel state information.

In spite of low complexity of DFT-based channel estimators, there is leakage when the multipaths are not exactly sample spaced [6], which induces an error floor for channel estimation. To reduce the error floor of DFT-based algorithm and increase estimation accuracy, more taps have 
to be used. Consequently, the estimation problem becomes ill-conditioned and noise may be enhanced. To improve the channel estimation accuracy for D-BLAST OFDM, we use optimum training sequences in $[5,7]$ not only for initial channel estimation but also for tracking channel autocorrelation matrix and then its dominant eigenvectors. The resultant eigenvectors are then used to form a transform which requires fewer taps to be estimated and reduces the error floor. The low-rank adaptive filter 1 (LORAF 1) in [8] is used for subspace tracking. For both proposed estimators, further refinement can be achieved by a robust filter [9] to exploit time-domain correlation.

The rest of this paper is organized as follows. In Section 2, we introduce D-BLAST OFDM systems. Then, in Section 3, we derive a layerwise LS channel estimator and analyze the mean square error (MSE) performance. Next, in Section 4, we propose an improved channel estimator based on subspace tracking. In Section 5, we evaluate the performance of a D-BLAST OFDM system with different channel estimation algorithms by computer simulation and major results of the paper are summarized in Section 6.

\section{D-BLAST OFDM SYSTEM}

Before introducing the channel estimation algorithm, we briefly describe D-BLAST for MIMO-OFDM in this section.

The complex baseband representation of a delay spread channel can be expressed as [10]

$$
h(t, \tau)=\sum_{l} \alpha_{l}(t) \Delta\left(\tau-\tau_{l}\right),
$$

where $\alpha_{l}(t)$ 's are wide-sense stationary narrowband complex Gaussian processes and are assumed to be independent among different paths. The channel may vary from block to block but stays the same within each OFDM block, which means that the effect of intercarrier interference (ICI) is not considered. Moreover, we assume the same normalized timedomain correlation function for all paths, that is,

$$
E\left\{\alpha_{l}(t+\Delta t) \alpha_{m}^{*}(t)\right\}= \begin{cases}\sigma_{l}^{2} r_{t}(\Delta t), & l=m, \\ 0, & l \neq m .\end{cases}
$$

Without loss of generality, we assume the total average power of the channel impulse response to be unity, that is,

$$
\sum_{l} \sigma_{l}^{2}=1
$$

For a MIMO-OFDM system with $N_{t}$ transmit and $N_{r}$ receive antennas $\left(N_{r} \geq N_{t}\right)$, the received signal at the $k$ th subcarrier of the $n$th block from the $j$ th receive antenna can be expressed as

$$
x_{j}[n, k]=\sum_{i=1}^{N_{t}} H_{i j}[n, k] b_{i}[n, k]+w_{j}[n, k],
$$

for $j=1, \ldots, N_{r}$ and $k=0, \ldots, K-1$, where $K$ is the total number of subcarriers of OFDM, $b_{i}[n, k]$ is the symbol transmitted from the $i$ th transmit antenna at the $k$ th subcarrier of the $n$th block, $H_{i j}[n, k]$ is the channel's frequency response at the $k$ th subcarrier of the $n$th block corresponding to the $i$ th transmit and the $j$ th receive antenna, and $w_{j}[n, k]$ is additive (complex) Gaussian noise that is assumed to be independent and identically distributed (i.i.d.) with zero-mean and variance $\rho$.

Equation (4) can also be written in matrix form as

$$
\mathbf{x}[n, k]=\mathbf{H}[n, k] \mathbf{b}[n, k]+\mathbf{w}[n, k],
$$

where

$$
\begin{aligned}
& \mathbf{x}[n, k]=\left(\begin{array}{c}
x_{1}[n, k] \\
\vdots \\
x_{N_{r}}[n, k]
\end{array}\right) \\
& \mathbf{H}[n, k]=\left(\begin{array}{cccc}
H_{11}[n, k] & H_{21}[n, k] & \cdots & H_{N_{t} 1}[n, k] \\
H_{12}[n, k] & H_{22}[n, k] & \cdots & H_{N_{t}}[n, k] \\
\vdots & \cdots & \ddots & \vdots \\
H_{1 N_{r}}[n, k] & \cdots & \cdots & H_{N_{t} N_{r}}[n, k]
\end{array}\right) \text {, } \\
& \mathbf{b}[n, k]=\left(\begin{array}{c}
b_{1}[n, k] \\
\vdots \\
b_{N_{t}}[n, k]
\end{array}\right) \text {, } \\
& \mathbf{w}[n, k]=\left(\begin{array}{c}
w_{1}[n, k] \\
\vdots \\
w_{N_{r}}[n, k]
\end{array}\right) \text {. }
\end{aligned}
$$

D-BLAST is an effective MIMO technique [2] that has been originally developed for a single-carrier system with flat fading channel. In this paper, we will use this technique for a MIMO-OFDM system, which can be shown in Figure 1. From the figure, the set of all subcarriers in an OFDM block is divided into $N_{t}$ subsets, each with $L=K / N_{t}$ subcarriers. Each layer, composed of $N_{t}$ such subsets associated with different transmit antennas, is encoded and decoded independently. Note that each layer still has $K$ subcarriers, but different subcarriers may be associated with different transmit antennas. Layers starting at block $n$ are denoted as $L_{p}[n]$, $p=1,2, \ldots, N_{t}$. With some abuse of notations, $k$ is both the subcarrier index and the symbol index for each layer. Given the structure of D-BLAST OFDM, the received signal at each receive antenna is the superposition of the desired signal, the signals already detected in the previous layers, and those undetected.

The signal detection of D-BLAST MIMO-OFDM is also very similar to the original D-BLAST. Assume that layer $L_{p}[n], p=1,2, \ldots, N_{t}$, is to be detected. From (4) we have

$$
\begin{aligned}
x_{j}\left[g_{p}(n, k), k\right] & =H_{f_{p}(k), j}\left[g_{p}(n, k), k\right] b_{f_{p}(k)}\left[g_{p}(n, k), k\right] \\
& +\sum_{i=1}^{f_{p}(k)-1} H_{i j}\left[g_{p}(n, k), k\right] b_{i}\left[g_{p}(n, k), k\right] \\
& +\sum_{i=f_{p}(k)+1}^{N_{t}} H_{i j}\left[g_{p}(n, k), k\right] b_{i}\left[g_{p}(n, k), k\right] \\
& +w_{j}\left[g_{p}(n, k), k\right],
\end{aligned}
$$




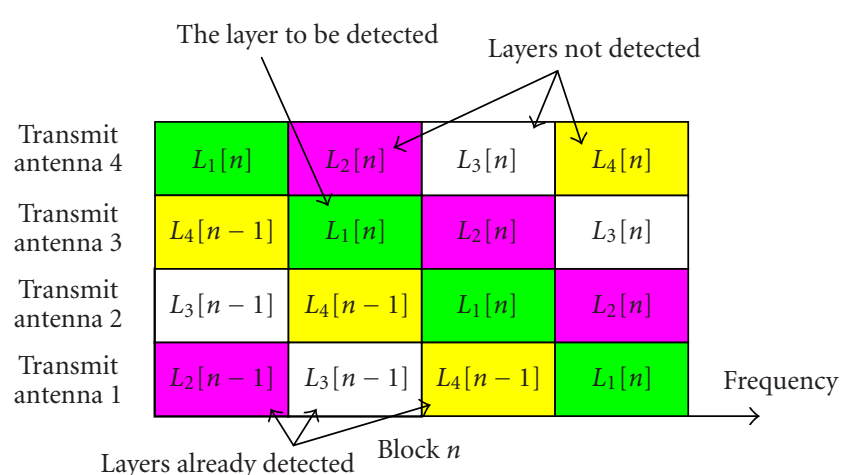

Layers already detected Block $n$

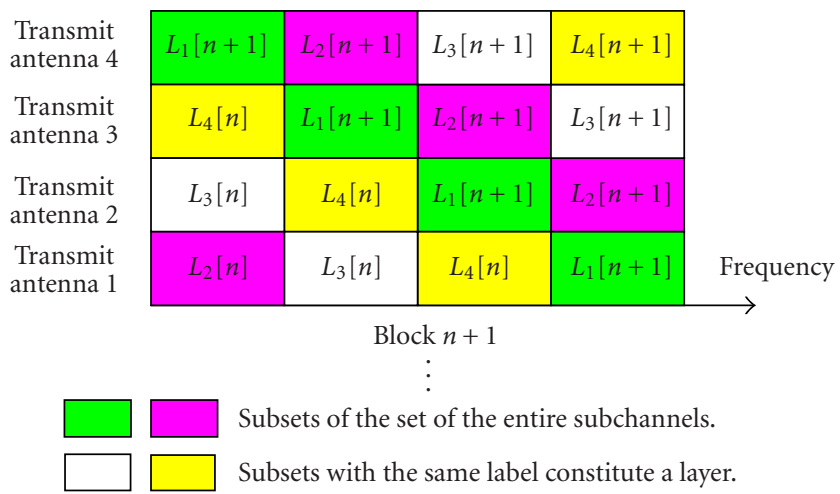

Figure 1: D-BLAST MIMO-OFDM structure.

for $j=1,2, \ldots, N_{r}$ and $k=0, \ldots, K-1$, where $f_{p}(k)$ and $g_{p}(n, k)$ are associations of the $k$ th symbol of layer $L_{p}[n]$ with transmit antenna and OFDM block, respectively, that is, the $k$ th symbol of layer $L_{p}[n]$ is sent from the $f_{p}(k)$ th transmit antenna via the $k$ th subcarrier of the $g_{p}(n, k)$ th OFDM block. Note that, in general, a layer spans two consecutive OFDM blocks, thus $g_{p}(n, k)$ is either $n$ or $n+1$. Equation (7) can be written in matrix notation as

$$
\begin{aligned}
\mathbf{x}\left[g_{p}(n, k), k\right]= & \mathbf{H}_{f_{p}(k)}\left[g_{p}(n, k), k\right] b_{f_{p}(k)}\left[g_{p}(n, k), k\right] \\
& +\sum_{i=1}^{f_{p}(k)-1} \mathbf{H}_{i}\left[g_{p}(n, k), k\right] b_{i}\left[g_{p}(n, k), k\right] \\
& +\sum_{i=f_{p}(k)+1}^{N_{t}} \mathbf{H}_{i}\left[g_{p}(n, k), k\right] b_{i}\left[g_{p}(n, k), k\right] \\
& +\mathbf{w}\left[g_{p}(n, k), k\right],
\end{aligned}
$$

where $\mathbf{H}_{i}[n, k]$ is the $i$ th column of $\mathbf{H}[n, k]$. Signals from antennas 1 to $f_{p}(k)-1$ have been detected and those from antennas $f_{p}(k)+1$ to $N_{t}$ are yet to be detected.

First, interference cancellation is carried out by subtracting detected signals from the received signal:

$$
\begin{aligned}
\tilde{\mathbf{x}}_{p}\left[g_{p}(n, k), k\right]= & \mathbf{x}\left[g_{p}(n, k), k\right] \\
& -\sum_{i=1}^{f_{p}(k)-1} \mathbf{H}_{i}\left[g_{p}(n, k), k\right] \hat{b}_{i}\left[g_{p}(n, k), k\right],
\end{aligned}
$$

where $\hat{b}_{i}[n, k]$ 's are detected symbols. Then interference from undetected signals is suppressed by linear combination that yields the maximum signal-to-interference-plus-noise ratio (SINR). Let

$$
\tilde{\mathbf{H}}_{p}[n, k] \triangleq\left(\mathbf{H}_{f_{p}(k)+1}[n, k], \mathbf{H}_{f_{p}(k)+2}[n, k], \ldots, \mathbf{H}_{N_{t}}[n, k]\right),
$$

then from [11], we have the following weighting vector:

$$
\mathbf{v}_{p}[n, k]=\left(\tilde{\mathbf{H}}_{p}[n, k] \tilde{\mathbf{H}}_{p}^{H}[n, k]+\rho \mathbf{I}\right)^{-1} \mathbf{H}_{f_{p}(k)}[n, k] .
$$

Thus, if we assume Gaussian distribution for the residual interference plus noise, the maximum likelihood decoding of layer $L_{p}[n]$ is to find $\left\{\hat{b}_{f_{p}(k)}\left[g_{p}(n, k), k\right]\right\}$ that minimizes

$$
\begin{aligned}
& M\left(\left\{\hat{b}_{f_{p}(k)}\left[g_{p}(n, k), k\right] ; k=0,1, \ldots, K-1\right\}\right) \\
& =\sum_{k=0}^{K-1} \frac{1}{\mathbf{v}_{p}^{H}[m, k]\left(\tilde{\mathbf{H}}_{p}[m, k] \tilde{\mathbf{H}}_{p}^{H}[m, k]+\rho \mathbf{I}\right) \mathbf{v}_{p}[m, k]} \\
& \left.\quad \cdot\left|\mathbf{v}_{p}^{H}[m, k]\left(\tilde{\mathbf{x}}_{p}[m, k]-\mathbf{H}_{f_{p}(k)}[m, k] \hat{b}_{f_{p}(k)}[m, k]\right)\right|^{2}\right|_{m=g_{p}(n, k)}
\end{aligned}
$$

which can be solved by standard Viterbi algorithm when convolutional codes are used. From the above discussions, channel information is crucial for the signal detection of $\mathrm{D}$ BLAST MIMO-OFDM. Therefore, we focus on channel estimation in the paper.

\section{LAYERWISE CHANNEL ESTIMATION}

In this section, we develop a layerwise LS channel estimation algorithm and analyze its performance.

\subsection{Layerwise least-square channel estimation}

Due to layerwise detection in D-BLAST, usually only partial knowledge of the symbols transmitted from all transmit antennas at one OFDM block is available after decoding of each layer. To exploit the characteristics of D-BLAST structure, channel estimation is carried out each time a layer is detected. Since channel responses are independent among different transmit-receive antenna pairs, we consider the channel estimation for one particular receive antenna and omit the receive antenna subscript $j$ in (4) to get

$$
x[n, k]=\sum_{i=1}^{N_{t}} H_{i}[n, k] b_{i}[n, k]+w[n, k] .
$$

After detection of layer $L_{p}[n]$, we estimate the channel responses at the $n$th block. Since only part of all the subcarriers of the current OFDM block have signals from all transmit antennas detected, we replace the received signals at subcarriers not fully detected with those of the previous OFDM block to form a complete received signal vector, due to the fact that 
$H_{i}[n, k] \approx H_{i}[n-1, k]$. Define

$$
\begin{aligned}
& z^{(p)}[n, k]= \begin{cases}x[n-1, k], & k \in \Sigma^{(p)}, \\
x[n, k], & \text { else, }\end{cases} \\
& \hat{d}_{i}^{(p)}[n, k] \triangleq \begin{cases}\hat{b}_{i}[n-1, k], & k \in \Sigma^{(p)}, \\
\hat{b}_{i}[n, k], & \text { else, }\end{cases}
\end{aligned}
$$

where $\Sigma^{(p)}=\left\{k ; g_{p}(n, k)=n\right.$ and $\left.f_{p}(k)<N_{t}\right\}$ is the set of subcarriers with signals not fully detected.

It is observed that with some leakage [6], channel frequency response can be approximated as

$$
H_{i}[n, k]=\sum_{l=0}^{\chi-1} h_{i}[n, l] W_{K}^{k l},
$$

where $W_{K}=e^{-j(2 \pi / K)}, \chi \geq\left\lceil t_{d} / t_{s}\right\rceil, t_{d}$ is the maximum delay spread and $t_{s}$ is the sampling interval which is equal to $1 / K \Delta f$ with $\Delta f$ being the tone spacing.

Let

$$
\begin{aligned}
& \mathbf{z}^{(p)}[n]=\left(z^{(p)}[n, 0], \ldots, z^{(p)}[n, K-1]\right)^{T}, \\
& \hat{\mathbf{D}}_{i}^{(p)}[n]=\operatorname{diag}\left\{\hat{d}_{i}^{(p)}[n, 0], \ldots, \hat{d}_{i}^{(p)}[n, K-1]\right\}, \\
& \mathbf{U}=\left(\begin{array}{cccc}
1 & 1 & \ldots & 1 \\
1 & W_{K} & \ldots & W_{K}^{\chi-1} \\
\vdots & \ldots & \ddots & \vdots \\
1 & W_{K}^{(K-1)} & \ldots & W_{K}^{(\chi-1)(K-1)}
\end{array}\right), \\
& \hat{\mathbf{h}}^{(p)}[n] \triangleq\left(\hat{\mathbf{h}}_{1}^{(p)^{T}}[n], \hat{\mathbf{h}}_{2}^{(p)^{T}}[n], \ldots, \hat{\mathbf{h}}_{N_{t}}^{(p)^{T}}[n]\right)^{T}, \\
& \hat{\mathbf{h}}_{i}^{(p)}[n] \triangleq\left(\hat{h}_{i}^{(p)}[n, 0], \ldots, \hat{h}_{i}^{(p)}[n, \chi-1]\right)^{T} .
\end{aligned}
$$

The LS channel estimation is to minimize the following cost function [4]:

$$
\begin{aligned}
& C\left(\left\{\hat{\mathbf{h}}_{i}^{(p)} ; i=1,2, \ldots, N_{t}-1\right\}\right) \\
& \quad=\left\|\mathbf{z}^{(p)}[n]-\sum_{i=1}^{N_{t}} \hat{\mathbf{D}}_{i}^{(p)}[n] \mathbf{U} \hat{\mathbf{h}}_{i}^{(p)}[n]\right\|^{2} .
\end{aligned}
$$

Then

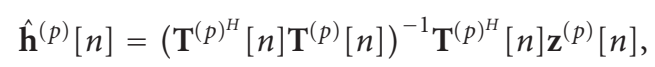

where

$$
\mathbf{T}^{(p)}[n]=\left(\hat{\mathbf{D}}_{1}^{(p)}[n] \mathbf{U}, \hat{\mathbf{D}}_{2}^{(p)}[n] \mathbf{U}, \ldots, \hat{\mathbf{D}}_{N_{t}}^{(p)}[n] \mathbf{U}\right) .
$$

The above estimate is further refined by applying a robust estimator for OFDM systems in [9], which makes full use of the correlation of channel parameters at different OFDM blocks.

\subsection{Performance analysis}

Here, we briefly analyze the performance of the above channel estimator for D-BLAST OFDM. Let

$$
\begin{aligned}
& \Delta \mathbf{h} \triangleq \mathbf{h}[n]-\mathbf{h}[n-1], \\
& \hat{s}_{i}^{(p)}[n, k] \triangleq \begin{cases}\hat{b}_{i}[n-1, k], & k \in \Sigma^{(p)}, \\
0, & \text { else, }\end{cases} \\
& \hat{\mathbf{S}}_{i}^{(p)}[n]=\operatorname{diag}\left\{\hat{s}_{i}^{(p)}[n, 0], \ldots, \hat{s}_{i}^{(p)}[n, K-1]\right\},
\end{aligned}
$$

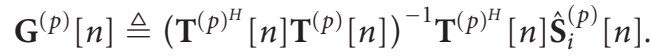

The MSE of the channel estimator is

$$
\begin{aligned}
\operatorname{MSE}^{(p)}[n] \triangleq & \frac{1}{N_{t} \chi} E\left\{\left\|\hat{\mathbf{h}}^{(p)}[n]-\mathbf{h}[n]\right\|^{2}\right\} \\
= & \frac{1}{N_{t} \chi} \mathbf{T r}\left\{\rho\left(\mathbf{T}^{(p)^{H}}[n] \mathbf{T}^{(p)}[n]\right)^{-1}\right. \\
& \left.\quad+\mathbf{G}^{(p)}[n] E\left(\Delta \mathbf{h}[n] \Delta \mathbf{h}^{H}[n]\right) \mathbf{G}^{(p)^{H}}[n]\right\},
\end{aligned}
$$

where $E\{\cdot\}$ denotes expected value of a random variable. Clearly the first term in the above equation results from noise and the second term is due to channel variation.

From the above discussion, the MSE of the channel estimate depends on the inverse of $\mathbf{T}^{(p)^{H}}[n] \mathbf{T}^{(p)}[n]$, which relates to the condition number of $\mathbf{T}^{(p)^{H}}[n] \mathbf{T}^{(p)}[n]$. It can be proved in the appendix using the bordering theorem for Hermitian matrices [12] that condition number of $\mathbf{T}^{(p)^{H}}[n] \mathbf{T}^{(p)}[n]$ increases with $\chi$. It implies that the channel estimation becomes more ill-conditioned as the number of parameters to be estimated increases. Thus we should choose the number of parameters as small as possible while preserving energy of the channel response, which is the reason for tracking the optimum transform matrix $\mathbf{U}$ in Section 4.

\section{SUBSPACE TRACKING}

The major problem of decision-directed channel estimation is the randomness of the symbol sequences during data transmission mode. For example, when the symbol sequences from any two of the transmit antennas are the same or very close, it is impossible or very hard to distinguish channel responses corresponding to different transmit antennas. The greater the number of transmit antennas, the more likely the channel is unidentifiable, or the more illconditioned channel identification is. Furthermore, to reduce the leakage of decision-directed DFT-based channel estimation in MIMO-OFDM systems, the number of taps representing channel frequency response has to be increased, which will make channel identification more ill-conditioned at the same time, as shown in Section 3. Moreover, increasing the number of taps makes the inverse operation of matrices in (22) more complicated. Hence, it is essential for lowcomplexity and high-performance channel estimator to reduce the number of parameters to be estimated while preserving most of the energy of channel frequency responses 
during the data transmission mode. Therefore, we will develop subspace tracking approaches to estimate channel parameters. And since the subspace only depends on channel autocorrelation matrix, which is time-invariant or changing very slowly, we apply subspace tracking only to training blocks and use the derived transform matrix instead of $\mathbf{U}$ defined in (18) for channel estimation during data transmission mode.

Let the $K \times K$ channel autocorrelation matrix $\left(\mathbf{R}_{f}\right)_{k_{1}, k_{2}}=$ $E\left\{H\left[k_{1}\right] H^{*}\left[k_{2}\right]\right\}$ have singular value decomposition as follows:

$$
\mathbf{R}_{f}=\mathbf{U}_{f} \Lambda \mathbf{U}_{f}^{H},
$$

where $\mathbf{U}_{f}$ is a $K \times K$ unitary matrix and $\Lambda=\operatorname{diag}\left\{\lambda_{1}\right.$, $\left.\lambda_{2}, \ldots, \lambda_{K}\right\}, \lambda_{1} \geq \lambda_{2} \geq \cdots>0$. From [13], optimum rank- $\chi$ estimator is to select eigenvectors $\mathbf{u}_{1}, \mathbf{u}_{2}, \ldots, \mathbf{u}_{\chi}$ corresponding to the $\chi$ biggest eigenvalues. Then the optimum rank- $\chi$ transform matrix is

$$
\mathbf{U}_{\mathrm{opt}}=\left(\mathbf{u}_{1}, \mathbf{u}_{2}, \ldots, \mathbf{u}_{\chi}\right) .
$$

Therefore, channel autocorrelation matrix is needed here for the optimum low-rank channel estimation.

To obtain the channel autocorrelation matrix, first we have to separate channel responses $H_{i}[n, k]$ 's for different $i$ 's. This can be done by appropriately designing the training block. In $[5,7]$, optimal training sequences have been proposed to maximally separate frequency responses of different transmit antennas while preserving most of the energy of each channel response. The training sequences are

$$
b_{i}[n, k]=b_{1}[n, k] W_{K}^{-K_{0}(i-1) k},
$$

for $i=1,2, \ldots, N_{t}$, where $K_{0}=\left\lfloor K / N_{t}\right\rfloor \geq\left\lceil t_{d} / t_{s}\right\rceil$ is the number of taps used to represent the channel response as a DFT transform. During the training period, we choose $K_{0}$ taps in approximating the channel response according to (15). Since the leakage introduced by the DFT-based approximation is decreased as $K_{0}$ increases and the well-designed training sequences provide maximum separability, we can set $K_{0}$ to be big enough such that the leakage is negligible while introducing little aliasing between different channel responses [5]. The procedure to separate channel responses can be described in Algorithm 1.

The dimension $\chi$ of the subspace can be either determined by minimum description length (MDL) criterion [14] that is not accurate for low signal-to-noise ratio (SNR) or slow channel variation, or by the approach in $[13,15]$ which argues that the essential dimension of a random signal is about the product of the bandwidth and time interval of the signal plus one. We just choose the latter approach for its simplicity and effectiveness; therefore, $\chi=\left\lceil t_{d} / t_{s}\right\rceil$. Subspace tracking approach can be summarized, which is in Algorithm 2 modified LORAF 1 in [8].

It should be noted that the robust channel estimator depends only on the subspace spanned by the dominant (a) During each training block, $\eta[n, k]=x[n, k] \cdot b_{1}^{*}[n, k]$.

(b) Perform IFFT on $(\eta[n, 0], \eta[n, 1], \ldots, \eta[n, K-1])$ to get $(\zeta[n, 0], \zeta[n, 1], \ldots, \zeta[n, K-1])$.

(c) For the channel response of transmit antenna $i$, circularly left shift $(\zeta[n, 0], \zeta[n, 1], \ldots, \zeta[n, K-1])$ by $(i-1) K_{0}$ to get $\left(\zeta^{\prime}[n, 0], \zeta^{\prime}[n, 1], \ldots, \zeta^{\prime}[n, K-1]\right)$. Let $\zeta^{\prime \prime}[n, k] \triangleq \begin{cases}\zeta^{\prime}[n, k], & k \in\left[0, K_{0}-1\right], \\ 0, & \text { else. }\end{cases}$

(d) A channel estimate $\hat{H}_{i}[n, k]$ is obtained by performing FFT on $\left(\zeta^{\prime \prime}[n, 0], \zeta^{\prime \prime}[n, 1], \ldots, \zeta^{\prime \prime}[n, K-1]\right)$.

Algorithm 1: Channel separation using the optimum training sequences.

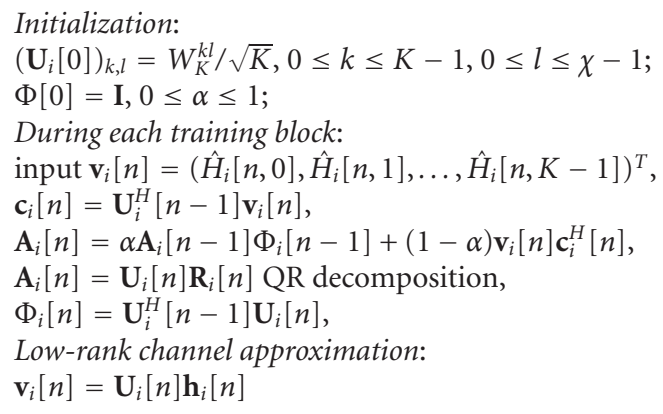

Algorithm 2: Subspace tracking for channel estimation.

eigenvectors rather than the particular eigenvectors. Let

$$
\hat{\mathbf{U}}_{i}[n]=\mathbf{U}_{\mathrm{opt}} \mathbf{Q}_{i}[n],
$$

where $\mathbf{Q}_{i}[n]$ is a $\chi \times \chi$ unitary matrix which accounts for the change of dominant eigenvectors without changing the subspace. Substituting (29) into (25), it can be easily verified that the MSE of the channel estimator is invariant to rotation of the dominant eigenvectors, which can also be seen in [9]. Therefore, it is the dominant subspace spanned by channel frequency responses that affects the performance of the subspace tracking-based channel estimator.

\section{SIMULATION RESULTS}

In this section, we evaluate the performance of different decision-directed channel estimation algorithms for DBLAST OFDM by computer simulation. Typical-urban (TU) channel with Doppler frequency $f_{d}=40$ and $200 \mathrm{~Hz}$ is used in our simulation. Performance of the proposed 7-tap layerwise subspace tracking estimator is simulated. As a comparison, performances of systems with ideal channel parameters, 7-tap layerwise estimator with optimum transform, as defined in (27), and 10-tap layerwise DFT-based estimator with significant tap selection (STS) [4] are evaluated. The performance of the traditional 10-tap blockwise DFT-based channel estimator is also given, where channel estimation is carried out once per OFDM block and the estimated channel parameters are used for the detection of the next OFDM block. 
Four transmit antennas and four receive antennas are employed to form four D-BLAST layers. Channel parameters corresponding to different transmit and receive antenna pairs are assumed to be independent but have the same statistics. The system bandwidth of $1.25 \mathrm{MHz}$ is divided into $256 \mathrm{sub}-$ channels: 2 subchannels on each side are used as guard tones, and the rest of the subchannels are used for data transmission. The symbol duration is $204.8 \mu \mathrm{s}$ and another $20.2 \mu \mathrm{s}$ is added as cyclic prefix (CP), resulting in a total block duration of $225 \mu \mathrm{s}$. A 16-state binary-to-4-ary convolutional codes of rate $1 / 2$ with the octal generators being $(26,37)[16]$ is used to encode the information bits in each layer. Four tail bits are used for trellis termination, leaving 248 information bits per layer. The encoder output is interleaved before sending to a transmit antenna at a particular subcarrier.

In each independent simulation, 2000 OFDM blocks of data are transmitted with 1 training block sent every 10 blocks. The performance averaged over independent simulations is evaluated. For channel estimator with subspace tracking, the first 50 blocks use 10-tap DFT-based estimator with STS. The estimated channel parameters are used for initial subspace acquisition so that initial training overhead can be saved at the expense of negligible performance loss for continuous data transmission. Then channel estimation is switched to the estimator with subspace tracking and the subspace is updated at each training block. The forgetting factor $\alpha$ is chosen to be 0.995 .

Figures $2 \mathrm{a}$ and $2 \mathrm{~b}$ compare the word error rate (WER) and bit-error-rate (BER) performance of different channel estimation algorithms when Doppler frequency is $40 \mathrm{~Hz}$. Of all estimators, the blockwise DFT-based channel estimator has the worst performance since it uses channel state information at the previous OFDM block for detection and thus is most susceptible to channel variation. The blockwise DFT-based estimator requires about $1 \mathrm{~dB}$ more SNR than the layerwise DFT-based estimator for a WER of $10^{-2}$ and its WER curve levels off quickly at high SNR's since its performance is bounded by channel variation. Among layerwise estimators, the subspace tracking estimator, requires $0.7 \mathrm{~dB}$ less SNR than the DFT-based estimator for $10^{-3}$ WER. Figure 2c shows how MSE evolves as the layerwise channel estimation progresses. From the figure, we can see that for all layerwise channel estimation methods, the most significant MSE improvement is seen after detection of the first layer of the current OFDM block, which is $0.7 \mathrm{~dB}$ at $\mathrm{SNR}=16 \mathrm{~dB}$, compared with about $0.16 \mathrm{~dB}$ per layer improvement for layers detected later with the proposed subspace tracking channel estimator.

For $f_{d}=200 \mathrm{~Hz}$, from Figure 3 we see that the performance difference between the blockwise channel estimator and layerwise estimators is even bigger now that the system performance is dominated by fast variation of channel parameters. The SNR gain for using layerwise subspace tracking estimator is about $0.8 \mathrm{~dB}$ for $10^{-2}$ WER compared with layerwise DFT-based estimator. It is clear that as the channel variation rate increases, the MSE performance improvement with layerwise channel estimation becomes more significant, with the successive MSE improvements being $3.4 \mathrm{~dB}, 1.2 \mathrm{~dB}$,

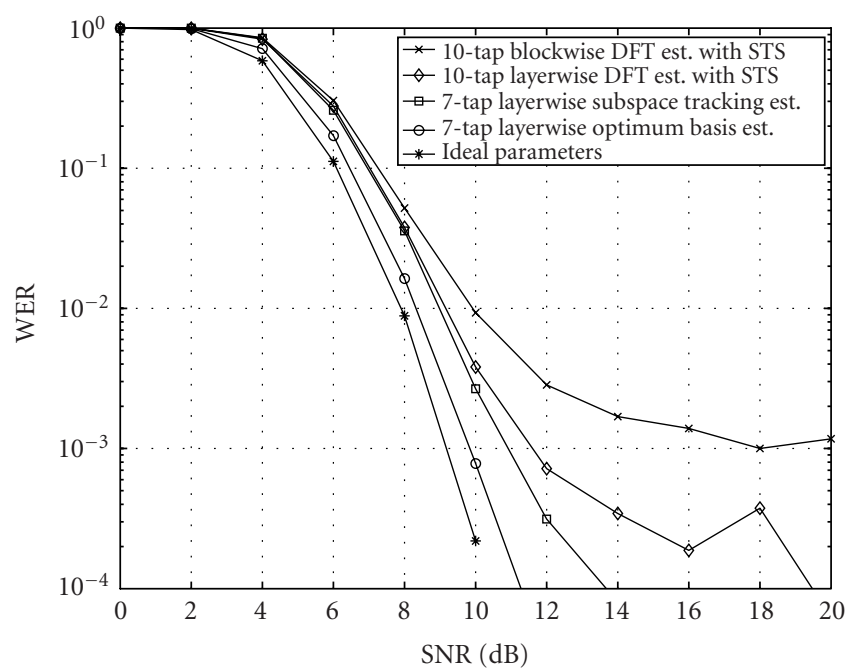

(a)

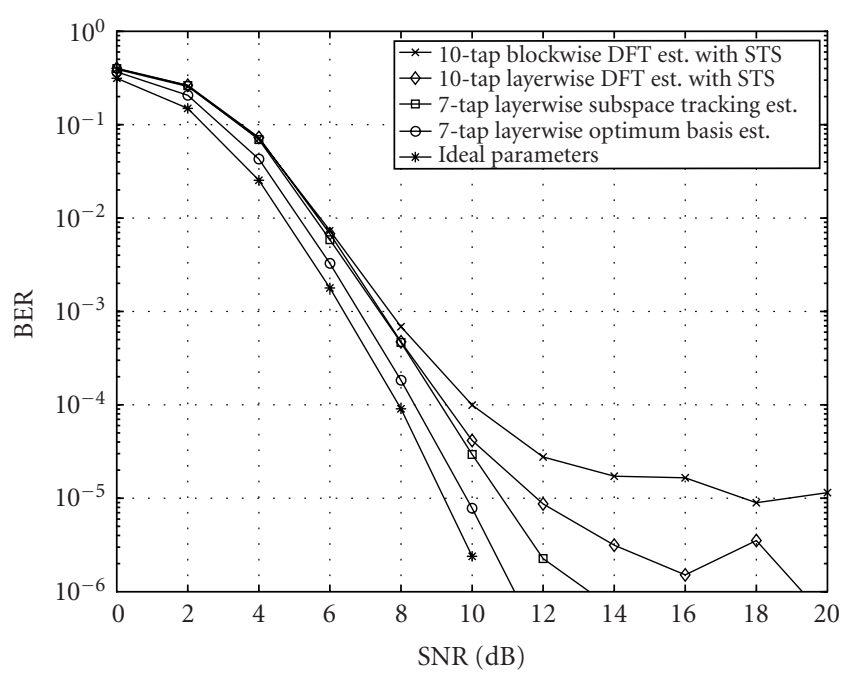

(b)

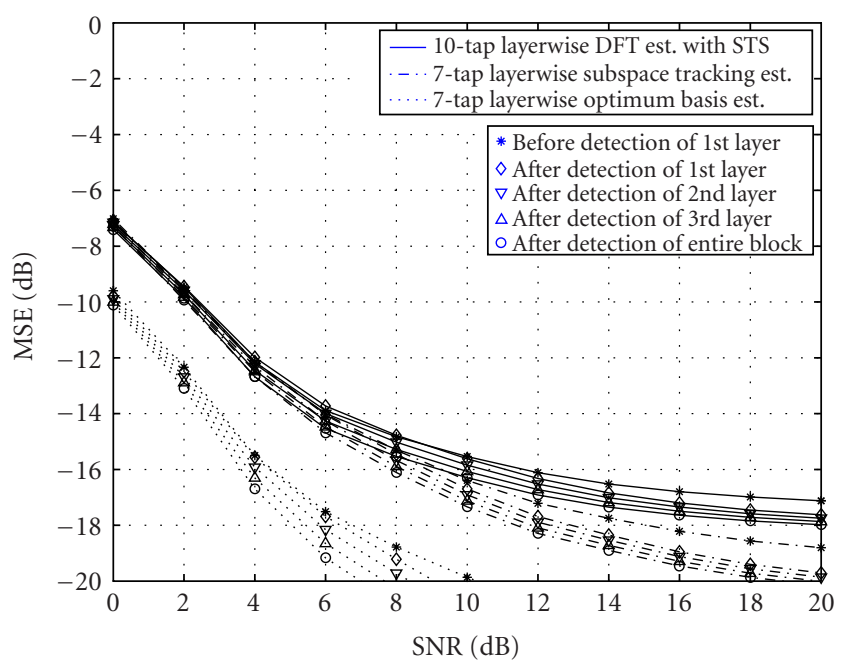

(c)

Figure 2: (a) WER, (b) BER, and (c) MSE of D-BLAST systems for channels with TU delay profile and $f_{d}=40 \mathrm{~Hz}$. 


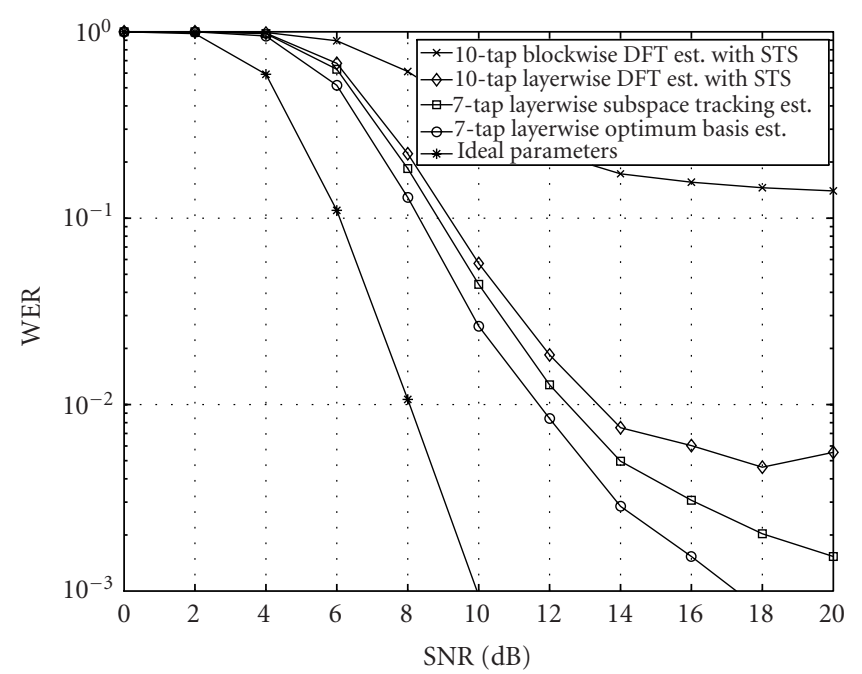

(a)

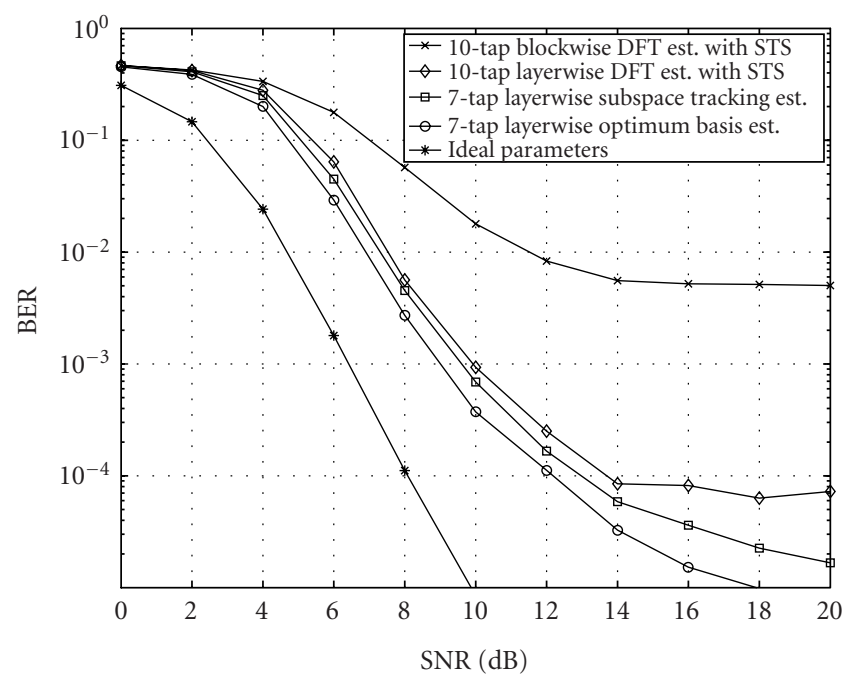

(b)

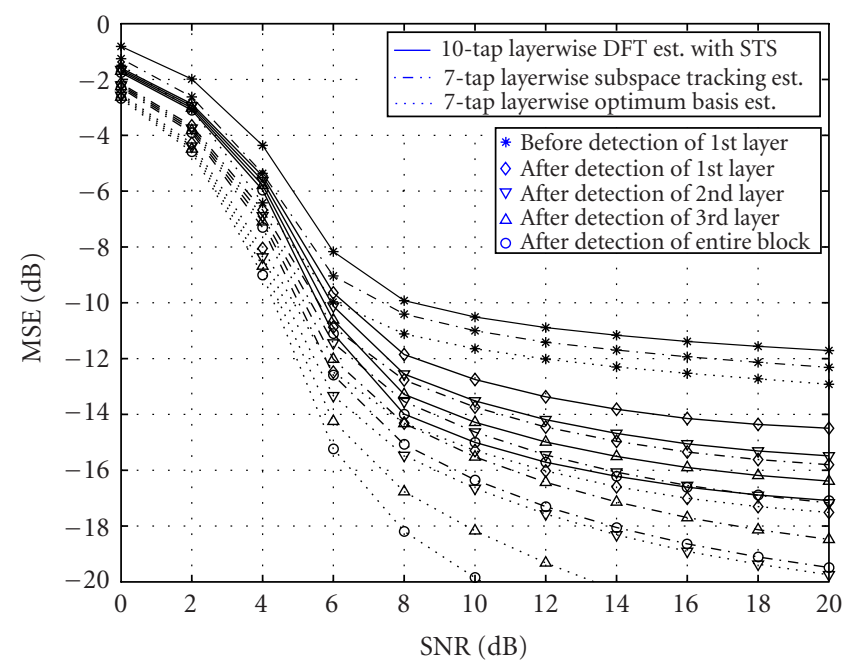

(c)

Figure 3: (a) WER, (b) BER, and (c) MSE of D-BLAST systems for channels with TU delay profile and $f_{d}=200 \mathrm{~Hz}$.
$1.2 \mathrm{~dB}$, and $1 \mathrm{~dB}$ at $\mathrm{SNR}=16 \mathrm{~dB}$, as observed in Figure 3c. For both $f_{d}=40$ and $200 \mathrm{~Hz}$, the subspace tracking estimator can effectively reduce the error floor thus provide better performance than that of the DFT-based estimator.

\section{CONCLUSION}

MIMO-OFDM is a promising technology that embraces advantages of both MIMO system and OFDM, that is, immunity to delay spread as well as huge transmission capacity. In this paper, we apply the D-BLAST structure to MIMOOFDM systems and develop a channel estimator that updates the estimated channel parameters in a layerwise fashion. Since we update channel estimation using detected signals to improve detection of the rest of the signals in the current OFDM block, the system is more robust to fast fading channels when compared with the traditional blockwise estimator. To further reduce the channel estimation error, we use the training blocks not only for channel estimation, but also for tracking of the dominant subspace spanned by the channel frequency response to reduce the number of parameters to be estimated during data transmission mode. Thus, additional performance improvement is obtained by using subspace tracking for the layerwise estimator, which is about $0.8 \mathrm{~dB}$ for $10^{-2}$ WER with $f_{d}=200 \mathrm{~Hz}$.

\section{APPENDIX}

Let $\mathbf{U}^{\prime}=\left(\mathbf{U}, \mathbf{u}_{\chi+1}\right)$, and from $\mathbf{U}^{\prime}$, we define $\mathbf{T}^{(p)^{\prime}}[n]$ as in (23) by substituting $\mathbf{U}^{\prime}$ for $\mathbf{U}$. We will show that

$$
\operatorname{cond}\left(\mathbf{T}^{(p)^{\prime H}}[n] \mathbf{T}^{(p)^{\prime}}[n]\right) \geq \operatorname{cond}\left(\mathbf{T}^{(p)^{H}}[n] \mathbf{T}^{(p)}[n]\right),
$$

where cond $(\cdot)$ means condition number of a matrix.

Proof. Let the eigenvalues of $\mathbf{T}^{(p)^{H}}[n] \mathbf{T}^{(p)}[n]$ be $\gamma_{1} \geq \gamma_{2} \geq$ $\cdots \geq \gamma_{\chi N_{t}}>0$. From (23) and by exchanging columns which does not change the eigenvalues, we have

$$
\mathbf{T}^{(p)^{\prime}}[n]=\left(\mathbf{T}^{(p)}[n], \mathbf{Y}^{(p)}[n]\right),
$$

where

$$
\begin{aligned}
& \mathbf{Y}^{(p)}[n]=\left(\hat{\mathbf{D}}_{1}^{(p)}[n] \mathbf{u}_{\chi+1}, \ldots, \hat{\mathbf{D}}_{N_{t}}^{(p)}[n] \mathbf{u}_{\chi+1}\right),
\end{aligned}
$$

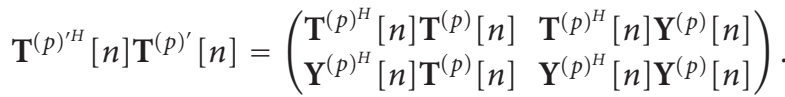

Let the eigenvalues of $\mathbf{T}^{(p)^{\prime H}}[n] \mathbf{T}^{(p)^{\prime}}[n]$ be $\gamma_{1}^{\prime} \geq \gamma_{2}^{\prime} \geq \cdots \geq$ $\gamma_{(\chi+1) N_{t}}^{\prime}>0$. By the bordering theorem for Hermitian matrices [12], we have

$$
\gamma_{1}^{\prime} \geq \gamma_{1} \geq \gamma_{\chi N_{t}} \geq \gamma_{(\chi+1) N_{t}}^{\prime}>0,
$$

thus

$$
\text { cond } \begin{aligned}
\left(\mathbf{T}^{(p)^{\prime H}}[n] \mathbf{T}^{(p)^{\prime}}[n]\right) & =\frac{\gamma_{1}^{\prime}}{\gamma_{(\chi+1) N_{t}}^{\prime}} \geq \frac{\gamma_{1}}{\gamma_{\chi N_{t}}} \\
& =\operatorname{cond}\left(\mathbf{T}^{(p)^{H}}[n] \mathbf{T}^{(p)}[n]\right) .
\end{aligned}
$$




\section{ACKNOWLEDGMENT}

This work was jointly supported by the National Science Foundation (NSF) under Grant CCR-0121565 and Nortel networks.

\section{REFERENCES}

[1] V. Tarokh, N. Seshadri, and A. R. Calderbank, "Space-time codes for high data rate wireless communication: performance criterion and code construction," IEEE Transactions on Information Theory, vol. 44, no. 2, pp. 744-765, 1998.

[2] G. J. Foschini, "Layered space-time architecture for wireless communication in a fading environment when using multielement antennas," Bell Labs Tech. Journal, vol. 1, no. 2, pp. 41-59, 1996.

[3] J. X. Du and Y. G. Li, "Channel estimation for D-BLAST OFDM systems," in Proc. IEEE Global Telecommunications Conference, vol. 1, pp. 335-339, Taipei, Taiwan, November 2002.

[4] Y. Li, N. Seshadri, and S. Ariyavisitakul, "Channel estimation for OFDM systems with transmitter diversity in mobile wireless channels," IEEE Journal on Selected Areas in Communications, vol. 17, no. 3, pp. 461-471, 1999.

[5] Y. Li, "Simplified channel estimation for OFDM systems with multiple transmit antennas," IEEE Transactions on Wireless Communications, vol. 1, no. 1, pp. 67-75, 2002.

[6] J. J. van de Beek, O. Edfors, M. Sandell, S. K. Wilson, and P. O. Borjesson, "On channel estimation in OFDM systems," in IEEE 45th Vehicular Technology Conference (VTC '95), vol. 2, pp. 815-819, Chicago, Ill, USA, July 1995.

[7] I. Barhumi, G. Leus, and M. Moonen, "Optimal training sequences for channel estimation in MIMO OFDM systems in mobile wireless channels," in Proc. IEEE International Zurich Seminar on Broadband Communications, Access, Transmission, Networking, pp. 44/1-44/6, Zurich, Switzerland, February 2002.

[8] P. Strobach, "Low-rank adaptive filters," IEEE Trans. Signal Processing, vol. 44, no. 12, pp. 2932-2947, 1996.

[9] Y. Li, L. J. Cimini, and N. R. Sollenberger, "Robust channel estimation for OFDM systems with rapid dispersive fading channels," IEEE Transactions on Communications, vol. 46, no. 7, pp. 902-915, 1998.

[10] R. Steele, Mobile Radio Communications, IEEE Press, New York, NY, USA, 1992.

[11] R. A. Monzingo and T. W. Miller, Introduction to Adaptive Arrays, John Wiley \& Sons, New York, NY, USA, 1980.

[12] R. A. Horn and C. R. Johnson, Matrix Analysis, Cambridge University Press, Cambridge, UK, 1985.

[13] O. Edfors, M. Sandell, J. J. van de Beek, S. K. Wilson, and P. O. Borjesson, "OFDM channel estimation by singular value decomposition," IEEE Transactions on Communications, vol. 46, no. 7, pp. 931-939, 1998.

[14] M. Wax and T. Kailath, "Detection of signals by information theoretic criteria," IEEE Trans. Acoustics, Speech, and Signal Processing, vol. 33, no. 2, pp. 387-392, 1985.

[15] H. J. Landau and H. O. Pollak, "Prolate spheroidal wave functions, fourier analysis and uncertainty-III: the dimension of the space of essentially time- and band-limited signals," Bell System Technical Journal, vol. 41, pp. 1295-1336, 1962.

[16] W. E. Ryan and S. G. Wilson, "Two classes of convolutional codes over GF( $q$ ) for $q$-ary orthogonal signaling," IEEE Transactions on Communications, vol. 39, no. 1, pp. 30-40, 1991.
Jianxuan Du obtained his B.S. and M.S. degrees in electrical engineering in 1998 and 2001, respectively, from Xi'an Jiaotong University, China. Since 2001, he has been pursuing the Ph.D. degree in electrical and computer engineering at Georgia Institute of Technology, Ga. He is currently a Research Assistant in Information Transmission and Processing Laboratory at Georgia Institute of Technology. His research interests include signal processing for wireless communications, channel estimation, and MIMO-OFDM systems.

Ye (Geoffrey) $\mathbf{L i}$ received his B.S.E. and M.S.E. degrees in 1983 and 1986, respectively, from the Department of Wireless Engineering, Nanjing Institute of Technology, Nanjing, China, and his Ph.D. degree in 1994 from the Department of Electrical Engineering, Auburn University, Alabama. After spending several years at AT\&T Labs Research, he joined Georgia Tech as an Associate Professor in 2000. His general re-

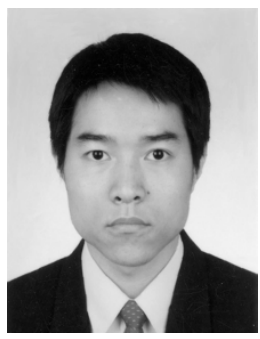
search interests include statistical signal processing and wireless communications. In these areas, he has contributed over 100 papers published in referred journals and presented in various international conferences. He also has over 10 USA patents granted or pending. He once served as a Guest Editor for two special issues on Signal Processing for Wireless Communications for the IEEE J-SAC. He is currently serving as an Editor for Wireless Communication Theory for the IEEE Transactions on Communications and an Editorial Board Member of EURASIP Journal on Applied Signal Processing. He organized and chaired many international conferences, including Vice-Chair of IEEE 2003 International Conference on Communications. 